\title{
How to begin to overcome the ambiguity present in differentiation between contents and levels of consciousness?
}

\author{
Talis Bachmann* \\ Laboratory of Cognitive Neuroscience, Institute of Public Law, University of Tartu, Tallinn, Estonia
}

\author{
Edited by: \\ Anil K Seth, University of Sussex, UK \\ Reviewed by: \\ Morten Overgaard, Aalborg \\ University, Denmark \\ ${ }^{*}$ Correspondence: \\ Talis Bachmann, Laboratory of \\ Cognitive Neuroscience, Faculty of \\ Law, Institute of Public Law, \\ University of Tartu (Tallinn branch), \\ Kaarli puiestee 3, Tallinn 10119, \\ Estonia. \\ e-mail: talis.bachmann@ut.ee
}

Recently, a welcome trend has emerged - in addition to the traditional studies on contents and states of consciousness, levels of consciousness have become a matter of research. However, there are some conceptual and methodological difficulties with this research - the labels used for empirical measurement of levels are ambiguous and underspecified while the research on neural correlates of consciousness has not been well linked to psychophysical approaches to studying the levels of consciousness. This article suggests a perspective on how to advance the psychophysics of measuring the levels by precisely specifying level-specific contents and how to relate the distinction between contents and levels to the distinction between the underlying brain mechanisms necessary for processing contents and regulating the level of consciousness.

Keywords: consciousness, contents, levels, neural correlates, states

\section{INTRODUCTION}

In terms of direct phenomenal experience, living subjects can be in two alternative basic states - the conscious and the unconscious state. The unconscious state cannot be characterized by its phenomenal contents because there are none; the examples include dreamless NREM sleep, coma, or states of being fainted or knocked out. Conscious state has phenomenal contents by default, characterized by variable qualia present in direct experience and appearing to a subject with variable "vividness" or "clarity." The basic conscious aware/awake state also varies along a continuum of levels, specified as a position on a scale above unconsciousness e.g., from dream consciousness in REM sleep, drowsiness, up to fully alert awake consciousness (Laureys and Tononi, 2009; Seth et al., 2011). The levels have been typically defined by objective indices of arousal and responsiveness where a mixture of objective physiological measurements and behavioral responses by subjects has been used; contents are defined by subjects' reports (Laureys, 2005; Laureys and Tononi, 2009; Hudetz and Pearce, 2010; Cavanna et al., 2011).

In Figure 1 the views of these approaches are illustrated in a generic simplified form. However, the approach illustrated in Figure $\mathbf{1}$ includes an inconsistency: state dependent levels are typically measured by objective means $\left(y_{\mathrm{obj}}\right)$, but level-specific conscious experiences have to be measured by subjective introspective means $\left(y_{\text {subj }}\right)$. The $x$-axis is typically left for contents. As one axis cannot represent two substantially different kinds, we need another axis - the $z$-axis for subjective measures of levels. We do this by "taking out" the "hidden" $y_{\text {subj }}$ from the $y$-axis of levels, leaving it exclusively for $y_{\mathrm{obj}}$. Now, the $y-z$ correspondences can be established by research where objective measures and signatures $y$ will be related to the corresponding subjective measures of level, $z$. For example, a NREM sleep signature of EEG specifying a point of value low on $y$-axis could find a correspondence in a near-zero point of value on $z$ (e.g., extremely rare, negligible dream experiences). Steady EEG occipital alphasuppression/beta enhancement signature having a relatively high $y$ value could correspond to a relatively high value $z$ (e.g., clear and vivid waking awareness of some scene). But even this approach is not complete because it is impossible to evaluate and measure subjective levels without measuring subjective contents of consciousness (awareness; Overgaard and Overgaard, 2010). The level-specific experiences $z$ inevitably have their qualitative contents $x$. Correspondences $z-x$ should be established. Figure 2 illustrates this.

The scientific approach to consciousness has been concerned with finding neural correlates of consciousness (NCC) either in terms of correlates of its contents $(x)$ or in terms of correlates of general states (operationalized as levels $y$ here). Unfortunately, the third dimension (subjective levels $z$ ) has remained largely ignored. Yet, if we have to measure objective counterparts of some subjective kinds, these subjective kinds have to be measurable as well. This applies not only to subjective contents $(x)$, but to subjective levels $(z)$ as well. Recently, a welcome trend has surfaced trying to integrate or combine these approaches, including addition of the concept of levels of consciousness to the specialist vocabulary and development of relevant methods for its measurement (Ramsøy and Overgaard, 2004; Overgaard et al., 2006; Hohwy, 2009; Dienes et al., 2010; Overgaard and Overgaard, 2010; Seth, 2010). Informed intuitions have lead to development of abstract computational approaches for measuring levels with the help of the concepts of causal density and integrated information (e.g., Balduzzi and Tononi, 2008; Seth et al., 2011). It seems not difficult in principle to find an empirical basis for testing the fit between the predictions about the level obtained from these computations and brain-process signatures (m/EEG, fMRI) associated with different behavioral levels. This 

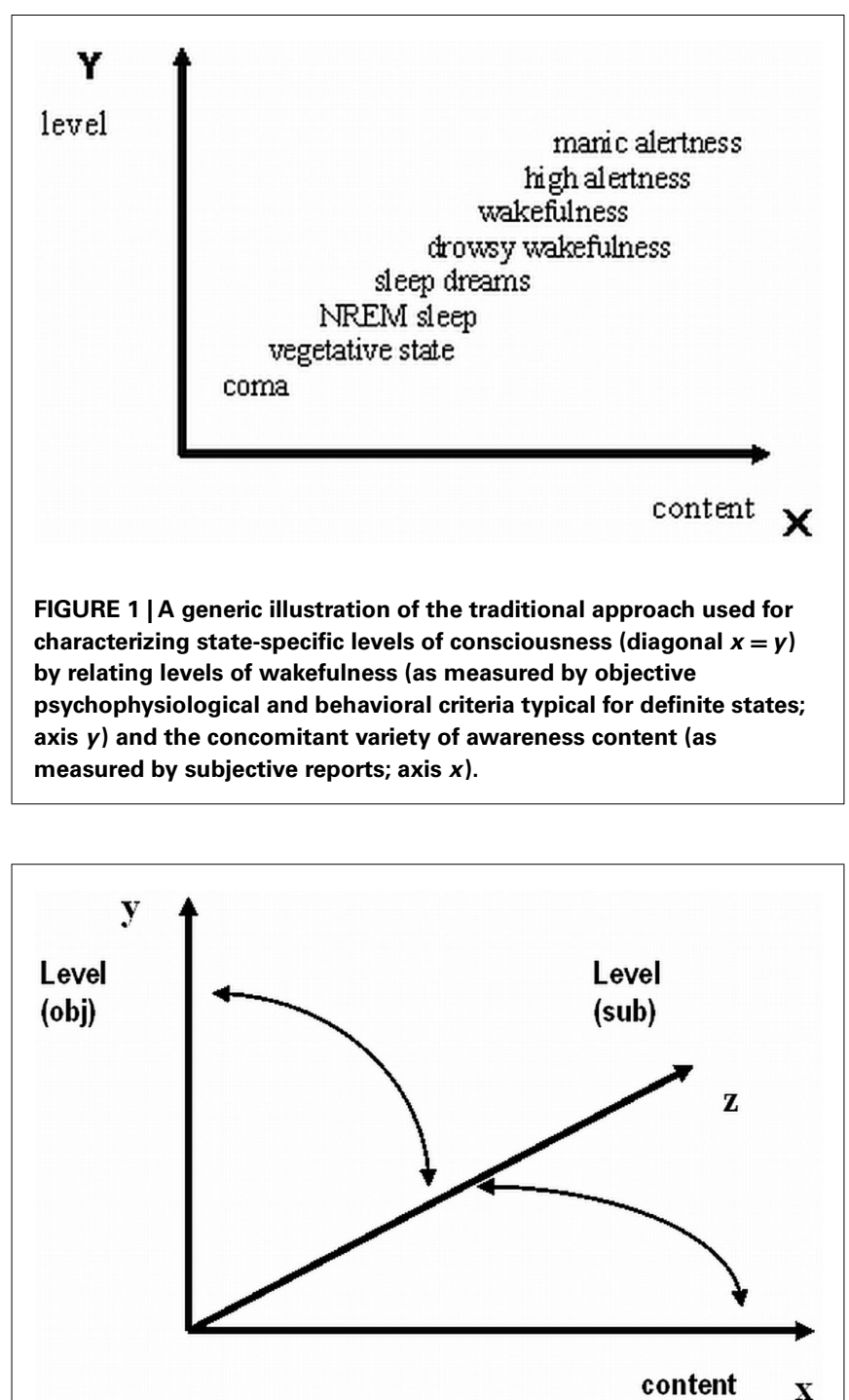

FIGURE 2 | Main correspondences between objective measures of level of consciousness $(y)$, subjective measures of level of consciousness $(z)$ and measures of content $(\boldsymbol{x})$. (The projection $y-x$ is omitted for parsimony, but it is potentially relevant as well).

means using $y$-axis for assessing correspondences between actual real levels and computed abstract measures of levels. However, a major problem is how to find scientifically sound methods for measurement of the subjective phenomenal levels $(z)$. I will point out some reasons why this problem persists, suggest where some progress could be made and conclude with the notion that and how identifying and combining neural mechanisms specialized for processing contents and for regulating levels may be a valuable approach.

\section{ASSESSMENT OF STATES, LEVELS, AND CONTENTS}

As stated above, researchers suggest to use an additional basic concept - the levels of consciousness, to be used for characterizing a richer continuum between fully unconscious and fully conscious states (e.g., Seth et al., 2008; Hohwy, 2009; Overgaard and Overgaard, 2010). In the clinical context there is a long tradition to evaluate levels of consciousness with the help of differentiation of physiological-behavioral states such as using the Glasgow coma scale (Teasdale and Jennett, 1974; Sleigh et al., 2010) or other methods (e.g., consult Laureys and Tononi, 2009). Because clinical methods have been "behavioristic," they have a limited use when we want to study levels of phenomenal consciousness and do this also in experimental situations with impoverished stimuli. Thus, the above mentioned trend to use the concept of subjective levels is indeed valuable.

Both contents and levels are assumed to be expressed in different degrees of clarity or degradation of subjective experience (Ramsøy and Overgaard, 2004; Overgaard and Overgaard, 2010). Projection of a position $y_{\mathrm{i}}$ onto a position $z_{\mathrm{i}}$ specifies an experience with a certain level of clarity typical for the objectively specified state-related level at that value of $y$; projection of a position $x_{i}$ onto a position $z_{\mathrm{i}}$ specifies an experience of certain content according to how it is characterized by a definite degree of clarity. But how can we measure degrees of subjective clarity? Historically, the tradition to measure degrees of perceptual consciousness goes back to the early microgenetic experiments (Bachmann, 2000). There, subjects in experiments rated their experiences of stimuli-objects by different adjectives corresponding to the different degrees of clarity - e.g., "something unspecified," "vague," "diffuse," "undifferentiated," "almost clear," "clear," etc. In a highly similar way, such gradations hoped to be helpful in characterizing varying levels are used also by modern researchers (e.g., Overgaard and Overgaard, 2010). Indeed, there is no other way to distinguish between the levels of consciousness than to use qualitatively different contents for this purpose (Overgaard and Overgaard, 2010). In terms of the present framework the subjective levels specified by a value on $z$ axis cannot be known unless characterized by a certain experience having its qualitative contents measurable along the $x$-axis. In this respect, the two approaches are consistent. Overgaards suggest that the "most clearly" experienced contents determines the present level of consciousness. Special introspection-based psychophysical methods of evaluation of the level of awareness through quantifiable degrees such as perceptual awareness scale (PAS) have been developed (Ramsøy and Overgaard, 2004; Sandberg et al., 2010). When PAS is used for evaluating the level of target stimulus' phenomenal experience, observers choose one of the four scale values in each trial of stimulus presentation - (1) No experience, (2) Brief glimpse, (3) Almost clear image, and (4) Absolutely clear image. There are already some promising results showing usefulness of PAS and other analogous scaling approaches for the measurement of the phenomenal level (e.g., Sandberg et al., 2011, found different dynamics of the awareness function and objective performance function with varying stimulation parameters). Yet, the ambiguity of the concepts used to describe and measure the levels $(z)$ continues to make obstacles for a satisfying development of the consciousness science. The main problem is that because level $(z)$ cannot be measured unless subject experiences certain contents $(x)$ corresponding to that level, there should be a satisfactory and well measurable grounding of the $z$-measures onto the $x$-measures. Up to now the empirically well founded $z$-to- $x$ projection is lacking. 


\section{PROBLEMS WITH MEASUREMENT OF LEVELS AND A SUGGESTED ADVANCEMENT}

Although introducing and productively using PAS (or other similar scales) is definitely a step forward, several problems remain.

First, if levels ( $z$ and/or $y$ ) are measured only through introspective contents $(x)$ by adjectives "clear," "almost clear," "weak glimpse," etc., it is difficult to use them reliably. These terms are (1) ambiguous (allowing variable interpretation) and (2) underspecified psychophysically (we do not know the objective equivalents in stimuli attributes on which evaluations are based). Although we agree that degrees of a level $(z)$ are specified indirectly with the help of some graded aspects of the subjective contents $(x)$, the adjectives used in PAS and other similar instruments bear too much uncertainty. Degrees vary with variations in the perceived features and feature values of the contents - brightness, contrast, fragmentariness, spatial frequency, sharpness/blur, etc. For example, the pictures of cats used by Overgaard and Overgaard, 2010, p. 2) for explaining clarity of perception can be psychophysically evaluated for their brightness/darkness, contrast, spatial frequency of detail. (See Figure 3 for examples from the Overgaards' article.) The precisely measurable psychophysical qualities should be made the basis of evaluation of the levels-bycontents in addition to or instead of the ambiguous and underspecified attributes such as the "almost clear," "clear," "vivid," etc. In other words, the positions on the $x$-axis have to be specified more precisely and with labels that have a well measurable and specific enough psychophysical attributes. The vague claritylabels can be decomposed to the labels that better correspond to the physical equivalents of measurement. Each subjective level must have its established objective stimulus-reference for content. For example, among the kitty pictures in Figure 3 the left one, when compared to the right one, appears to have less blur, higher contrast, lower overall apparent brightness, higher salience values of image elements, etc. Promising experimental methods for a more precise measurement of subjective levels include: forced choice among many objective examples potentially similar to the subjective percepts (e.g., variably contrasted, blurred, fragmented objective images); thorough qualitative interviews distilled into inter-subjective descriptive invariants; gathering overwhelmingly typical depictions (e.g., drawings) produced by independent observers as the standard objective equivalents for

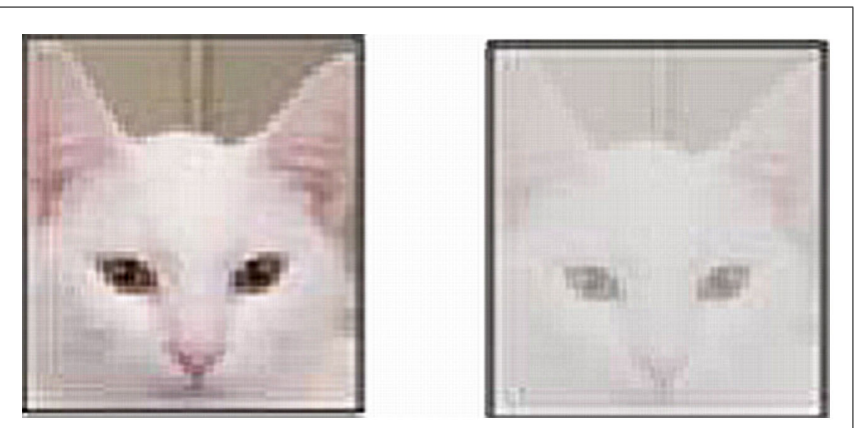

FIGURE 3 | Examples of pictures used by Overgaard and Overgaard (2010) to illustrate the perception according to different subjective levels of clarity. subjective experiences. Understandably, from the practical point of view this means additional work because for each stimulus environment used in a particular study (and with certain subjects) objective stimuli examples mimicking different subjective contents that correspond to different levels should have been prepared in preliminary experiments.

The principal method to "objectivize" the introspective phenomenal levels-by-contents of target stimulus awareness would consist in using objective stimuli-references for this purpose. We would find out the contents $\left(x_{\mathrm{i}}\right)$ of perceptual experience that correspond to a certain scale point of the level $\left(z_{\mathrm{i}}\right)$, but in order to do this in a less ambiguous and a well objectivized way we would produce objective depictions of the stimuli according to which ones among a prepared variety are evaluated as best correspondences to the perceived level-specific contents by the subjects. For example, as a first step, we can run preliminary studies with varying experimental conditions where subjects produce variablecontent drawings representative of subjective stimulus experience or where subjects participate in a forced choice procedure where they have to choose among a multitude of pre-set stimulus examples prepared as the likely representatives of subjective stimulus experience. Typical produced or prevailingly chosen objective counterparts (references) are found for each level of awareness and reference stimuli with considerable inter-observer correspondence will have been selected. Thereafter, as a second step, one can run main experiments where different subjects have to perceive the target stimuli again in varying conditions - consider variations in objective stimulation conditions such as exposure duration or contrast or variation in subjective state such as drowsiness, intoxication, wakeful alertness, etc. In a forced choice procedure subjects then choose between the objectivized reference stimuli prepared in the first stage of research and corresponding to the different levels-by-contents. If subjects often choose a reference that corresponds to some content associated with a lower level related experience, it can be concluded that level of conscious awareness in perceiving the target stimulus was relatively low. (Objective experimental means for obtaining variable levels of target stimuli experience include variable exposure durations, masking SOAs, contrast levels, attentional resource availability, etc. The subject-related means for obtaining or taking advantage of variable levels of target stimuli experience include timing from waking up, psycho-stimulant or -depressant use, treatment with anesthetics, etc.) Understandably, different modalities and domains of stimuli will have their own contents based specific measured out gradations of levels.

The second problem emerges when we ask again - consciousness of what? For instance, Overgaard and Overgaard (2010) specify the level "barely conscious" as "not characterized by any content" (p. 2). Suppose we find that the highest degree of target awareness under given circumstances is "something characterized by no contents." However, absence of experienced content for target stimuli (and impossibility to choose any reference stimulus offered) need not mean there is no subjective content at all. Non-target content can relate to the level of consciousness in different ways not necessarily specifying only one level of consciousness. Targets may not be perceived in their full-level or lower level consciousness for various reasons; non-perceived 
targets can be inferred by fully conscious subjects from the other, well experienced cues or by logical inferences. When subjects report "no experience" of targets they can guess, but guesses are founded on certain subjective experiential states dependent on expectancies, memory, vague intuitive feelings, etc. The partial awareness account (Kouider et al., 2010) argues that reported target stimulus awareness may result not only from the direct experience of the target as supported by exhaustive bottom-up processing, but also from the lower level conscious representation (of the partial information) insufficient for target experience in itself, but sufficient for subserving the top-down effects creating illusory conscious percepts. In this case the lower level target consciousness is associated with higher-level non-target consciousness. Based on the feeling of a "weak glimpse," a level of consciousness is termed "barely conscious," but it may be "fully conscious by a weak glimpse” instead. Consequently, PAS-like scales should be developed further by specifying them according to the objective criterion contents on which the clarity-evaluations are founded. Here again the above suggested procedures of objectivization by finding and using level-specific reference stimuli could help.

The third problem appears because an invariant physical stimulus can be perceived as having one or another content and associated with a varying degree of clarity. One-time perception of a stimulus undergoes a microgenetic percept formation with qualitatively different subjective contents alternating in real time (Bachmann, 2000). The level of consciousness of a stimulus progressively changes along the unfolding microgenesis. Consequently, various levels as being based on varying contents can be specified also within each single instance of target perception with invariant subject's state. We should distinguish whether the resulting contents based measurement of the level is determined by (1) the objective stimulus parameters, (2) the state of the subject, or (3) the stage the microgenesis of percept has reached, given invariant stimulus and state.

Cognition is prone to illusions and subjectively vivid nonveridicalities accompanied by high confidence (Bachmann, 2004; Kouider et al., 2010). Hence the fourth problem: reports on target clarity, even if fine-tuned with the help of objective reference stimuli, cannot be used as the only criteria with which we measure conscious contents and levels. It is useful to look for the objective brain-process signatures of subjective clarity and confidence, combined with the earlier mentioned behavioral methods of validation of levels of experience by reliable inter-subjective objectivization.

Up to now, brain-imaging of NCCs has been split between studying contents and studying states (Hohwy, 2009; Kouider et al., 2010). Distinction between content- and level-specific neural systems is scarce (e.g., Seth et al., 2008). Interactivity between neural systems specialized for contents and for level of expression of consciousness has been rarely used (e.g., Bachmann, 1984, 1999; Edelman and Tononi, 2000; Ribary, 2005). Although in terms of the subjective criteria used for specifying the levels we cannot overcome the contents-dependence of the "level" (Overgaard and Overgaard, 2010), we can objectively study interactivity and mutual influences between the content-specific brain systems and the level-regulating brain systems.

\section{BRAIN MECHANISMS USEFUL FOR PROCESSING CONTENTS AND REGULATING LEVELS}

Earlier, I have suggested distinguish between (1) brain mechanisms for pre-conscious and conscious cognitive-perceptual processing of contents and (2) brain mechanisms for modulation of the content-specific processes up to the level sufficient for conscious experience of these contents (Bachmann, 1984, 1999, 2000). Recently, Lamme (2010) presented a neural stance for differentiating four stages of processing as related to phenomenal consciousness. However, as Lamme's work is concerned primarily with the aspect of contents and does not consider level-related processes, I will not extend the discussion of Lamme's work in the context of the present paper. For example, Lamme's model lacks contribution of the non-specific reticulo-thalamic mechanisms (the system for regulating levels). His is a cortical process theory of conscious vs. unconscious processing. In my opinion, without incorporating the mechanisms of thalamo-cortical interaction objective differences between unconscious vs. conscious processing and between contents vs. levels of consciousness cannot be understood (Bachmann, 1984, 1999, 2000). In my view, the key to a successful approach for meaningfully relating the subjective contents $(x)$ and subjective levels $(z)$ is to ascertain if there are brain mechanisms specialized, accordingly, for processing the contents and regulating the levels.

When we supplement the introspective measurements by objective physiological measurements distinguishing between the contribution of the brain mechanisms for contents and brain mechanisms for levels, an opportunity emerges. This stance presupposes a possibility to distinguish between neural signatures (neural correlates, NC) associated with levels of processing and neural signatures (NC) associated with contents of processing. NC for the contents (NCcont) could be found such that they objectively and veridically discriminate between stimuli. (In terms of Kouider et al., 2010, this gives a premise either for unconscious, partially conscious or fully conscious access to the contents.) Overcoming the ambiguity of the acronym NCC (which stands for both the contents and the level of consciousness) we could term contents of consciousness as the contents of processing specified by NCcont, but also supplemented by additional neural processes $\mathrm{X}$ that exemplify the level of processing necessarily present in the conscious state. Stated simply, NCcont $+\mathrm{X}=\mathrm{NCC}$. This X is not only the factor responsible for processing at the level where the pre-conscious contents become conscious contents, but also the factor that allows contents to be expressed at a variable level, if modulations performed by X are incrementally leading to changes in the contents $(x)$ that stand for varying levels $(z)$. Most significantly, being able to measure contributions of NCcont to the levels-by-contents and contributions of $\mathrm{X}$ to the levels-by-contents separately we will have an additional means to differentiate and measure levels and contents from the same metatheoretical perspective and do this in a more objective way. Thus, an important direction of empirical research should be to find links between subjects' evaluations produced by advanced (i.e., more precisely objectivized) PAS-type scales and brain-process signatures indicative of relative involvement of NCcont and X.

Does this perspective have any scientific evidence in its support? I think the answer is affirmative. It is widely accepted that 
the level of consciousness (both in terms of $y$ and in terms of $z$ ) is a function of the extended reticulo-thalamo-cortical system that itself does not process the perceptual contents, but is capable of modulating how clear, non-fragmented, steady, and veridical the experience of contents is (Edelman and Tononi, 2000; Ribary, 2005; Laureys and Tononi, 2009; Hudetz and Pearce, 2010). This is a good candidate for being the mechanism X. It is important to notice that although the main function of the thalamo-cortical modulation system is to enable consciousness and modulate the specific contents-carrying cortical neurons, the thalamo-cortical complex does indeed specify content and level together. This is because the axons from this system project onto content-specific pyramidal neurons in the cortex and the effect of the modulating system inevitably manifests in contentful experiences. From the point of view of necessary ongoing research this means that we should disentangle and meaningfully relate brain processes indicative of the contribution of the modulating system and processes indicative of the contribution of the primary specific afference that (can) function(s) autonomously from $\mathrm{X}$.

Specific contents can be veridically processed by the specialized cortical modules even though the level of activity remains below the threshold needed for consciousness (Bachmann, 1999; Baars et al., 2003; Rose, 2006; Kouider et al., 2010). This represents the mechanisms that produce NCcont. In the perceptual retouch theory (Bachmann, 1999, 2000) cortical specific modules fulfill the function of NCcont. If non-specific thalamocortical modulation (the $\mathrm{X}$ ) of the nodes standing for NCcont becomes effective, the level of activity signified by NCcont is upgraded so that pre-conscious contents become conscious contents; NCcont $+\mathrm{X}=\mathrm{NCC}$. It is easy to see that NCC involves recorded processes indicative of interaction of the content-wise non-specific, level-regulating processes and the content-wise specific processes. In the dynamically changing product of the interactive workings of the mechanisms signified by NCcont and mechanisms of $\mathrm{X}$ both contents and level are integrated. In a nutshell, thalamo-cortical non-specific modulation regulates the level, which leads to microgenetically advancing integration of contents represented by cortical nodes (by which we can evaluate level), which all can be controlled by cortical top-down operations as the set of access mechanisms. It would be a nagging task to explore whether mechanism for NCcont serves primarily the function of conveying information, with $\mathrm{X}$ serving the function of integration. If this proves to be true, we will have a conceptualization harmonious with theories where levels of consciousness are a function of information being integrated (Balduzzi and Tononi, 2008; Seth et al., 2011).

At this point it is important to comment on the possibility of two types of content. It is possible that there are sensory-perceptual features that can be reliably represented by the contents-system unconsciously (i.e., information transmitted and stored without much loss even if the level-regulating system has not upgraded these representations to the phenomenally explicit level). Information is present, but it is not integrated with the conscious scene. For example, color, low-to-medium spatial frequency content, orientation, form, typical object configuration, an image of a threatening face, and some other types of content can be listed here. Then there may be other features and attributes that could be more intimately dependent on the influence of the level-regulating system, such as subjective contrast, high spatial frequency edges, fragmentariness/unity of experienced scene, temporal frequency of subjective change, etc. An important experimental task would be to try to differentiate between the features or attributes of contents that are highly sensitive to the modulation by the level system and features that can be represented invariantly beginning from the lowest level of consciousness up to its highest level. The task of disentangling NCC that are signatures of the contribution of the system $\mathrm{X}$ from the neural signatures of the contribution of the system that already pre-consciously can encode level-independent contents seems equally important. Here is a speculative example: it is possible that we find ERP or spectral perturbation signatures that are invariant to different levels, but signify encoding of certain visual-categorical contents invariantly with levels; vice versa - we may find signatures that are invariant to level-independent contents, but vary with varying levels. For example, fast ERP components that can be evoked also in sleeping or anesthetized subjects may stand for the former and N100-150 or slow negative potentials may stand for the latter. With invasive electrodes permitting LFP or single-cell recording the perspective of the above described strategy seems even better. (Consider firing of face-sensitive neurons independent of how vividly or whether consciously at all the subject processes a face stimulus while the level-determining systems' activity fluctuates. Alternatively, consider invariant firing of an intralaminar thalamic neuron whether the contents of conscious perception vary or not.)

Fully conscious experiences are not limited to perception of the environmental stimulation - consider dreams, hallucinations, spontaneous recollections. Compared to the psychophysical approach, the approach acknowledging (NCcont $+\mathrm{X})$ helps to objectivize the levels-by-contents of consciousness when endogenous experiences are the case. As people cannot imagine, dream, or hallucinate about anything that could not consist of the combination of the elements of phenomenal experiences founded on the learned objective stimulus attributes, it should be possible, in principle, to interpret the NCC for assessing the level. This can be based on the results of the earlier research on perception of the objective stimulation.

\section{CONCLUDING REMARKS}

In this paper, I have suggested two directions of research and theory in an attempt to advance measurement of levels of consciousness. First, there appears to be a need for methods that help to objectivize the introspectively founded scales used in evaluating the levels of conscious experience through the concomitant graded contents. Second, it seems useful to distinguish between brain mechanisms for contents of consciousness and for regulating the levels of consciousness. The described approach has merit because the known neurophysiology of unconscious as well as consciouslevel experience involves modular brain systems for representing specific content and being capable of working under different levels of consciousness (Baars et al., 2003; Rose, 2006). On the other hand, the reticulo-thalamic modulating system that itself does not carry information about the contents can upgrade the activity of the specific system so that its contents become represented at 
the phenomenal explicit level (Bachmann, 1999; Ribary, 2005). Thus, it was inviting to speculate that the distinctions between contents and levels (e.g., Overgaard and Overgaard, 2010) may be mechanistically founded, respectively, on the brain systems signifying NCcont and brain systems X. Becoming conscious of certain contents and varying the levels of conscious experience depends on interactive workings of the systems for NCcont and systems X. In a purely behavioral experiment it is never possible to distinguish the contributions of the contents- and levels-systems

\section{REFERENCES}

Baars, B., Banks, W., and Newman, J. (eds). (2003). Essential Sources in the Scientific Study of Consciousness. Cambridge, MA: MIT Press/Bradford Book.

Bachmann, T. (1984). The process of perceptual retouch: nonspecific afferent activation dynamics in explaining visual masking. Percept. Psychophys. 35, 69-84.

Bachmann, T. (1999). "Twelve spatiotemporal phenomena, and one explanation," in Cognitive Contributions to the Perception of Spatial and Temporal Events, eds G. Aschersleben, T. Bachmann, and J. Müsseler (Amsterdam: Elsevier), 173-206.

Bachmann, T. (2000). Microgenetic Approach to the Conscious Mind. Amsterdam: John Benjamins.

Bachmann, T. (2004). Inaptitude of the signal detection theory, useful vexation from the microgenetic view, and inevitability of neurobiological signatures in understanding perceptual (un)awareness. Conscious. Cogn. 13, 101-106.

Balduzzi, D., and Tononi, G. (2008). Integrated information in discrete dynamical systems: motivation and theoretical framework. PLoS Comput. Biol. 4, el000091. doi:10.1371/journal.pcbi.1000091

Cavanna, A. E., Shah, S., Eddy, C. M., Williams, A., and Rickard, H. (2011). Consciousness: a neurological perspective. Behav. Neurol. 24, 107-116.

Dienes, Z., Scott, R. B., and Seth, A. K. (2010). Subjective measures of implicit knowledge that go beyond confidence: reply to Overgaard et al. Conscious. Cogn. 19, 685-686.

Edelman, G., and Tononi, G. (2000). A Universe of Consciousness: How Matter Becomes Imagination. New York: Basic Books.

Hohwy, J. (2009). The neural correlates of consciousness: new experimental approaches needed? Conscious. Cogn. 18, 428-438.

Hudetz, A., and Pearce, R. (eds). (2010). Suppressing the Mind: Anesthetic Modulation of Memory and Consciousness. New York: Humana Press.

Kouider, S., de Gardelle, V., Sackur, J., and Dupoux, E. (2010). How rich is consciousness? The partial awareness hypothesis. Trends Cogn. Sci. (Regul. Ed.) 14, 301-307.

Lamme, V. A. F. (2010). How neuroscience will change our view on consciousness. Cogn. Neurosci. 1, 204-240.

Laureys, S. (2005). The neural correlate of (un)awareness: lessons from the vegetative state. Trends Cogn. Sci. (Regul. Ed.) 9, 556-559.

Laureys, S., and Tononi, G. (eds). (2009). The Neurology of Consciousness: Cognitive Neuroscience and Neuropathology. Amsterdam: Elsevier.

Overgaard, M., and Overgaard, R. (2010). Neural correlates of contents and levels of consciousness. Front. Psychol. 1:164. doi:10.3389/fpsyg.2010.00164

Overgaard, M., Rote, J., Mouridsen, K., and Ramsøy, T. Z. (2006). Is conscious perception gradual

whereas it seems realistic in principle to accomplish this distinction by brain-imaging and decomposing the NCcont $+\mathrm{X}$.

\section{ACKNOWLEDGMENTS}

Many thanks go to Anil Seth and two anonymous referees who substantially helped to advance this paper. Research presented here was supported by Estonian Scientific Competency Council (targeted financing research theme SF0182717s06, "Mechanisms of Visual Attention").

or dichotomous? A comparison of report methodologies during a visual task. Conscious. Cogn. 15, 700-708.

Ramsøy, T. Z., and Overgaard, M. (2004). Introspection and subliminal perception. Phenomenol. Cogn. Sci. 3, 1-23.

Ribary, U. (2005). Dynamics of thalamo-cortical network oscillations and human perception. Prog. Brain Res. 150, 127-142.

Rose, D. (2006). Consciousness. Oxford: Oxford University Press.

Sandberg, K., Bibby, B. M., Timmermans, B., Cleeremans, A., and Overgaard, M. (2011). Measuring consciousness: task accuracy and awareness as sigmoid functions of stimulus duration. Conscious. Cogn. 20, 1659-1675.

Sandberg, K., Timmermans, B., Overgaard, M., and Cleeremans, A. (2010). Measuring consciousness: is one measure better than the other? Conscious. Cogn. 19, 1069-1078.

Seth, A. K. (2010). The grand challenge of consciousness. Front. Psychol. 1:5. doi:10.3389/fpsyg.2010.00005

Seth, A. K., Barratt, A. B., and Barnett, L. (2011). Causal density and integrated information as measures of conscious level. Philos. Transact. A Math. Phys. Eng. Sci. 369, 3748-3767.

Seth, A. K., Dienes, Z., Cleeremans, A., Overgaard, M., and Pessoa, L. (2008). Measuring consciousness: relating behavioural and neurophysiological approaches. Trends Cogn. Sci. (Regul. Ed.)12, 314-321.
Sleigh, J., Steyn-Ross, M., Steyn-Ross, A., Voss, L., and Wilson, M. (2010). "Anesthesia-induced state transitions in neuronal populations," in Suppressing the Mind: Anesthetic Modulation of Memory and Consciousness, eds A. Hudetz and R. Pearce (New York: Humana Press), 139-160.

Teasdale, G., and Jennett, B. (1974). Assessment of coma and impaired consciousness. Lancet 2, 81-84.

Conflict of Interest Statement: The author declares that the research was conducted in the absence of any commercial or financial relationships that could be construed as a potential conflict of interest.

Received:06 November 2010; accepted: 02 March 2012; published online: 21 March 2012.

Citation: Bachmann T (2012) How to begin to overcome the ambiguity present in differentiation between contents and levels of consciousness? Front. Psychology 3:82. doi: 10.3389/fpsyg.2012.00082

This article was submitted to Frontiers in Consciousness Research, a specialty of Frontiers in Psychology.

Copyright () 2012 Bachmann. This is an open-access article distributed under the terms of the Creative Commons Attribution Non Commercial License, which permits non-commercial use, distribution, and reproduction in other forums, provided the original authors and source are credited. 